\title{
Effects of Ginseng on Fertility
}

\author{
Bo Hyon Yun ${ }^{1,2}$, Young Sik Choi ${ }^{1,2}$, SiHyun $\mathrm{Cho}^{1,2}$, Byung Seok Lee ${ }^{1,2}$, Si Kwan Kim ${ }^{3}$ and Seok Kyo Seo ${ }^{* 1,2}$ \\ ${ }^{1}$ Department of Obstetrics and Gynecology, Severance Hospital, Yonsei University College of Medicine, Republic of Korea \\ ${ }^{2}$ Institute of Women's Life Medical Science, Severance Hospital, Yonsei University College of Medicine, Seoul, Republic of Korea
}

${ }^{3}$ Department of Obstetrics and Gynecology, Gangnam Severance Hospital, Yonsei University College of Medicine, Seoul, Republic of Korea

Received: 踾: November 03, 2018; Published: 制: November 14, 2018

*Corresponding author: Seok Kyo Seo, M.D., Department of Obstetrics and Gynecology, Severance Hospital, Yonsei University College of Medicine, 50 Yonsei-ro, Seodaemun-gu 120-752, Seoul, Republic of Korea

\begin{abstract}
Ginseng is a representative, traditional Eastern Asian herb used as a tonic for slowing down the aging process. It has become one of the most popular dietary supplements throughout the world. The major active ingredient of ginseng is the ginseng saponin, which is composed of various ginsenosides. Currently, approximately 30 ginsenosides have been identified. Few research studies have attempted to identify the exact effects exerted by each type and constituent of ginseng. Interestingly, the induction of apoptosis of cancer cell lines has been reported in several malignancies, and effects on dopamine release pathways in the brain have been shown. In addition to the traditional medicinal property of ginseng, which has been thought to be a tonic, a few studies have been conducted to identify whether it can improve fertility. However, whether ginseng affects fertility or not remains experimental since most of the studies have been performed in vitro. The aim of this present study was to review relevant studies on the effect of ginseng on male and female reproduction, especially infertility.
\end{abstract}

Keywords: Female and Male Infertility; Fertility; Ginseng

Abbreviations: LH: Luteinizing Hormone; GABA: Gamma Aminobutyric Acid; GDNF: Glial Cell Derived Neurotrophic Factor; CAMP: Cyclic Adenosine Monophosphate; KRG: Korean Red Ginseng; PCOS: Polycystic Ovary Syndrome

\section{Introduction}

Ginseng is a representative, traditional Eastern Asian herb used as a tonic for slowing down the aging process [1]. The major active ingredient of ginseng is the ginseng saponin, which is composed of various ginsenosides. Currently, approximately 30 ginsenosides have been identified [2-4]. There are at least 9 known species of ginseng, including the Asian (Panax ginseng), American (Panax quinquefolium), and Japanese (Panax japonicus) ginseng. These varieties have shown effects such as antioxidant effects, enhancement of diverse physiologic effects including immunostimulatory effects $[5,6]$ effects on the neuronal system $[7,8]$ and control of the metabolic state [9-11]. In addition to the traditional properties of ginseng, which has been thought to be tonic, a few studies have been conducted to identify whether it can improve fertility. However, whether ginseng affects fertility or not and its underlying mechanisms of action remain to be investigated in humans since most related studies have been performed in vitro. The aim of this mini-review is to assess and highlight previous relevant studies of the effect of ginseng on male and female reproduction, especially fertility.

\section{Ginseng and Male Fertility}

The relationship between ginsenosides and male reproduction has been evaluated for over 20 years. However, few studies have been performed in humans, and most have used animal models. Ginseng has been considered a tonic, and therefore, a considerable number of experiments have been conducted to investigate its effects on sexual performance. In terms of libido, enhancement of copulatory behavior after treatment with Panax ginseng and Panax quinquefolium has been demonstrated in rodent models [12]. Not all varieties of ginseng have shown the advantage of increasing the sex drive in studies, which may have been caused by different components that may or may not increase serum testosterone and luteinizing hormone (LH) levels. In vitro studies on neurotransmitters such as dopamine, acetylcholine, and gamma aminobutyric acid (GABA) have also shown an association with ginsenoside [13-16]. Ginsenoside Rb1 treatment increased LH secretion in the anterior pituitary gland of male rats [17].

In terms of ginseng's effects on semen, numerous studies have shown that it improved semen quality in animal models. In 
rats, various ginsenosides have shown enhancing effects on sperm count and motility after treatment. Saponins from the cultured root of wild P. ginseng showed effects on spermatogenesis in male rats [18]. In the study, the researchers administered P. ginseng powder orally after inducing oligospermia in rats using dioxin and examined the sperm count and testes histologically. The P. ginseng saponin revived spermatogenesis in their study, suggesting its possible role in reversing the damage. In previous studies, ginseng-treated rats have shown increased spermatogenesis by increasing the glial cell-derived neurotrophic factor (GDNF) expression in Sertoli cells [19] and testicular cyclic adenosine monophosphate (cAMP)responsive element modulator (CREM) [20]. GDNF is known to be a possible regulating factor of the lifespan of spermatogonial cells, and CREM is an essential factor for spermatid maturation. An animal study using P. ginseng showed sperm hyperactivation at the genetic level, suggesting possible improvement of sperm quality [21]. In rat models of induced genito-urinary inflammation such as epididymo-orchitis and prostatitis, Korean Red ginseng (KRG) enhanced the anti-infective effects when it was administered with antibiotics. In addition, it increased sperm motility while decreasing apoptosis in testicular tissue and stimulating the yield of normal spermatozoa [22,23]. In their study performed in rats treated with cyclophosphamide and ginseng, Akram et al. [24] showed possible gonadoprotective effects of ginseng. They suggested that the possible protective role may be related to a ginseng-induced decrease in reactive oxygen species.

In human studies, ginseng has shown positive effects on sexual performance in men. In a double-blind, placebo-controlled study involving 45 men with erectile dysfunction, subjects showed an improvement in erectile performance and sexual satisfaction after treatment with KRG for 8 weeks [24]. Moreover, the beneficial effect of KRG on erection in men with erectile dysfunction has been identified in meta-analyses of randomized controlled trials [25]. In vitro studies showed increased nitric oxide synthesis in endothelial cells after treatment with ginsenosides [26,27], which has been suggested as a possible underlying mechanism for improving erectile dysfunction. However, although meta-analyses and in vitro studies showed the effectiveness of KRG, the exact mechanism remains largely unclear. However, diverse results have been shown in investigations of whether ginseng improves semen quality or not. When human semen was directly treated with ginseng extract in vitro, significant changes were observed in the motility and viability of the sperm [28]. In a case-control study, treatment with P. ginseng was reported to improve the sperm quality and sex hormone profiles [12]. However, the characteristics of the participants were diverse, and the number of participants was small. Recently, a randomized, double-blinded clinical study performed in 80 men with varicocele has shown significant improvement in semen parameters [29] after treatment with KRG for 12 weeks. However, this study also failed to show any differences in pre- and post-treatment serum hormonal levels.

Under disease conditions such as cancer and diabetes, and in the post-radiation period, ginseng showed improvement of sperm profiles after treatment of animals exposed to varying degrees of radiation [30] suggesting a future positive role for ginseng in this area. However, most of the data have been obtained from animal studies and not studies in humans. Moreover, there is a lack of knowledge about the effective dose recommended for humans, and studies evaluating the effects of ginseng on infertile couples or patients with male infertility, or pregnancy outcomes are lacking.

\section{Ginseng and Female Fertility}

Although a few studies have evaluated the effect of ginseng on the male reproductive system, much fewer studies have been conducted in female subjects. In the few studies conducted thus far, there has been emphasis on polycystic ovary syndrome. Polycystic ovary syndrome (PCOS) or polycystic ovary has been frequently associated with oligo-/anovulation, which causes difficulty in achieving pregnancy. A previous study delineating the changes in the structure of the ovarian theca internal cells following KRG administration in a rat model suggested that KRG possibly stimulated steroid-producing cells [31]. Subsequently, intraperitoneal infusion and oral administration of KRG improved the polycystic ovary morphology, with decreased neuronal growth factor expression in the ovaries, suggesting a possible role of KRG in PCOS $[1,32,33]$.

A study conducted by Zhu et al. [35] showed the protective effect of American ginseng in premature ovarian failure in rats. They selected molecular markers (PLA2G4A, miR-29a, and miR144) of ovarian function based on their previous study, and measured mRNA and protein expression as well as prostaglandin E2, LH, follicle-stimulating hormone, and estradiol levels before and after treatment with American ginseng [34,35]. The analysis of transcriptional and protein variations, as well as variations in hormone levels, suggested that American ginseng might have protected the ovaries against failure by regulating prostaglandin biosynthesis, ovulation, and preventing ovarian aging. In contrast, in a previous study, KRG saponins have shown significantly positive effects on oocyte quality in rats, decreasing the proportion of abnormal oocytes [36]. In that study, KRG saponins improved the hormonal profiles of female rats during the ovulatory cycle. Ginsenoside Rb1 significantly decreased preovulatory serum levels of androgens and 17-beta estradiol, and ginseng protopanaxadiol saponins significantly increased postovulatory serum progesterone levels. In addition to improving the oocyte quality, ginsenosides have been shown to stimulate antioxidant enzymes in rat embryos, which may lead to improvements in the quality of embryos [37]. Lee et al. showed a significant increase in mRNA expression levels of glutathione peroxidase after treatment with ginsenoside Rb1, Rc, and Re.

One of the most well-known effects of ginseng may be its role as a complementary medicine in perimenopausal women. A randomized study conducted in 384 Swedish postmenopausal women showed improvement in the quality of life but no improvement in vasomotor symptoms and hormonal profiles [38]. However, a randomized, double-blinded clinical trial conducted using KRG in 72 Korean peri- and post-menopausal women has 
shown significant improvements in vasomotor symptoms [39]. Moreover, in that study, KRG showed favorable changes in the markers of cardiovascular diseases, such as total cholesterol, low-density lipoprotein cholesterol, and carotid intima-media thickness. Until now, as a complementary medicine, ginseng was thought to mainly have a tonic effect, possibly improving vasomotor symptoms in postmenopausal women.

\section{Ginseng and Pregnancy Outcome}

The first report of the effects of ginseng on pregnancy outcome was published in 1990: it was a case report of neonatal androgenization with a mother who had used Siberian ginseng constantly during pregnancy to early breastfeeding [40]. In China, a study group reported the possible beneficial effect of ginseng use during pregnancy in mothers showing fetal intrauterine growth restriction. However, the case-control study was composed of a small number of participants [41]. Few studies have evaluated the effect or toxicities of ginseng since the early 1990s.

The investigation of the effect of ginseng on embryos has been focused on ginseng's antioxidative activity and potential teratogenicity. Because of ethical limitations, studies cannot be performed on human embryos and have only been performed in animals. The antioxidant effect of ginseng has been evaluated in alcohol-induced toxicity during pregnancy. For example, in embryo cultures of Japanese ricefish, co-culturing with P. ginseng has been shown to improve the effects of ethanol toxicity [42]. Black ginseng has been shown to recover antioxidant enzyme levels and inhibit teratogenesis induced by ethanol in mouse embryo cultures [43] However, despite the positive effects of ginseng, concerns have been raised over potential teratogenicity. Although no unfavorable effects on neonates of mothers who had consumed ginseng during pregnancy were noticed in a large birth cohort data [44] ginsenoside $\operatorname{Rg} 1$ has been proven to be embryotoxic in rat and mouse embryo developmental models [45]. Although ginseng has possible benefits for assisted reproductive technology or pregnancy, the available data on whether ginseng has positive or negative effects on embryonic development are limited.

\section{Conclusion}

Although ginseng is one of the most popular herbs consumed globally and specifically believed to have antifatigue, immunological, hormonal, and tonic effects, not many studies have been performed to investigate its reproductive effects. Considering the possible effects of ginseng on the hormone profiles of male and female subjects and its antioxidant property, further investigations of its application in reproductive medicine is expected.

\section{Acknowledgement}

This study was supported by Korea Institute of Planning \& Evaluation for Technology in Food, Agriculture, Forestry \& Fisheries, Korea (Grant number 113040-3).

\section{References}

1. Pak SC, Lim SC, Nah SY, Lee J, Hill JA, et al. (2005) Role of Korean red ginseng total saponins in rat infertility induced by polycystic ovaries. Fertil Steril 84(2): 1139-1143.
2. Kim DH, Moon YS, Lee TH, Jung JS, Suh HW, et al. (2003) The inhibitory effect of ginseng saponins on the stress-induced plasma interleukin-6 level in mice. Neurosci Lett 353(1): 13-16.

3. Tachikawa E, Kudo K, Hasegawa H, Kashimoto T, Sasaki K, et al. (2003) In vitro inhibition of adrenal catecholamine secretion by steroidal metabolites of ginseng saponins. Biochem Pharmacol 66(11): 22132221.

4. Bae EA, Han MJ, Kim EJ, Kim DH (2004) Transformation of ginseng saponins to ginsenoside Rh2 by acids and human intestinal bacteria and biological activities of their transformants. Arch Pharm Res 27(1): 6167.

5. Bak MJ, Hong SG, Lee JW, Jeong WS (2012) Red ginseng marc oil inhibits iNOS and COX-2 via NFkappaB and p38 pathways in LPS-stimulated RAW 264.7 macrophages. Molecules 17(12): 13769-13786.

6. Lin QY, Jin LJ, Cao $\mathrm{ZH}, \mathrm{Xu}$ YP (2008) Inhibition of inducible nitric oxide synthase by Acanthopanax senticosus extract in RAW264.7 macrophages. J Ethnopharmacol 118(2): 231-236.

7. Ye R, Yang Q Kong X, Han J, Zhang X, et al. (2011) Ginsenoside Rd attenuates early oxidative damage and sequential inflammatory response after transient focal ischemia in rats. Neurochem Int 58(3): 391-398.

8. Nah SY, Bhatia KS, Lyles J, Ellinwood EH, Lee TH (2009) Effects of ginseng saponin on acute cocaine-induced alterations in evoked dopamine release and uptake in rat brain nucleus accumbens. Brain Res 1248: 184-190.

9. DeSouza LR, Jenkins AL, Jovanovski E, Rahelić D, Vuksan V (2015) Ethanol extraction preparation of American ginseng (Panax quinquefolius $L$ ) and Korean red ginseng (Panax ginseng C.A. Meyer): differential effects on postprandial insulinemia in healthy individuals. J Ethnopharmacol 159: 55-61.

10. Lian F, Wu L, Tian J, Jin M, Zhou S, et al. (2015) The effectiveness and safety of a danshen-containing Chinese herbal medicine for diabetic retinopathy: a randomized, double-blind, placebo-controlled multicenter clinical trial. J Ethnopharmacol 164: 71-77.

11. Zhang E, Gao B, Yang L, Wu X, Wang Z, et al. (2016) Notoginsenoside Ft1 Promotes Fibroblast Proliferation via PI3K/Akt/mTOR Signaling Pathway and Benefits Wound Healing in Genetically Diabetic Mice. J Pharmacol Exp Ther 356(2): 324-332.

12. Salvati G, Genovesi G, Marcellini L, Paolini P, De Nuccio I, et al. (1996) Effects of Panax Ginseng C.A. Meyer saponins on male fertility. Panminerva Med 38(4): 249-254.

13. Benishin CG (1992) Actions of ginsenoside Rb1 on choline uptake in central cholinergic nerve endings. Neurochem Int 21(1): 1-5.

14. Kimura T, Saunders PA, Kim HS, Rheu HM, Oh KW, et al. (1994) Interactions of ginsenosides with ligand-bindings of $\operatorname{GABA}(\mathrm{A})$ and GABA(B) receptors. Gen Pharmacol 25(1): 193-199.

15. Yuan CS, Attele AS, Wu JA, Liu D (1998) Modulation of American ginseng on brainstem GABAergic effects in rats. J Ethnopharmacol 62(3): 215222.

16. Shi J, Xue W, Zhao WJ, Li KX (2013) Pharmacokinetics and dopamine/ acetylcholine releasing effects of ginsenoside Re in hippocampus and mPFC of freely moving rats. Acta Pharmacol Sin 34(2): 214-220.

17. Tsai SC, Chiao YC, Lu CC, Wang PS (2003) Stimulation of the secretion of LH by Ginsenoid Rb1 in rats. Chin J of Physiol 46(1): 1-7.

18. Park JS, Hwang SY, Lee WS, Yu KW, Paek KY, et al. (2006) The therapeutic effect of tissue cultured root of wild Panax ginseng C.A. Mayer on spermatogenetic disorder. Arch Pharm Res 29(9): 800-807.

19. Yang WM, Park SY, Kim HM, Park EH, Park SK, et al. (2011) Effects of Panax ginseng on glial cell-derived neurotrophic factor (GDNF) expression and spermatogenesis in rats. Phytother Res 25(2): 308-311. 
20. Park WS, Shin DY, Kim DR, Yang WM, Chang MS, et al. (2007) Korean ginseng induces spermatogenesis in rats through the activation of cAMPresponsive element modulator (CREM). Fertil Steril 88(4): 1000-1002.

21. Park EH, Kim DR, Kim HY, Park SK, et al. (2014) Panax ginseng induces the expression of genes and sperm hyperactivation. Asian J Androl 16(6): 845-851.

22. Eskandari M, Ghalyanchi Langeroudi A, Zeighami H, Rostami A, Kazemi M, et al. (2016) Co-administration of ginseng and ciprofloxacin ameliorates epididymo-orchitis induced alterations in sperm quality and spermatogenic cells apoptosis following infection in rats. Andrologia $49(3)$.

23. Miri M, Shokri S, Darabi S, Alipour Heidari M, Ghalyanchi A, et al. (2016) Efficacy of Compound Therapy by Ginseng and Ciprofloxacin on Bacterial Prostatitis. Cell J 18(1): 103-111.

24. Hong B, Ji YH, Hong JH, Nam KY, Ahn TY, et al. (2002) A double-blind crossover study evaluating the efficacy of korean red ginseng in patients with erectile dysfunction: a preliminary report. J Urol 168(5): 20702073.

25. Jang DJ, Lee MS, Shin BC, Lee YC, Ernst E, et al. (2008) Red ginseng for treating erectile dysfunction: a systematic review. Br J Clin Pharmacol 66(4): 444-450.

26. Chen X, TJ Lee (1995) Ginsenosides-induced nitric oxide-mediated relaxation of the rabbit corpus cavernosum. Br J Pharmacol 115(1): 1518.

27. Murphy LL, TJ Lee (2002) Ginseng, sex behavior, and nitric oxide. Ann N Y Acad Sci 962:372-377.

28. Liu J, Liang P, Yin C, Wang T, Li H, et al. (2004) Effects of several Chinese herbal aqueous extracts on human sperm motility invitro. Andrologia 36(2): 78-83.

29. Park HJ, Choe S, Park NC (2016) Effects of Korean red ginseng on semen parameters in male infertility patients: A randomized, placebocontrolled, double-blind clinical study. Chin J Integr Med 22(7): 490-495.

30. Leung KW, AS Wong (2013)Ginseng and male reproductive function. Spermatogenesis 3(3): e26391.

31. Rim BM (1979) Ultrastructural studies on the effects of Korean Panax ginseng on the theca interna of rat ovary. Am J Chin Med 7(4): 333-344.

32. Jung JH, Park HT, Kim T, Jeong MJ, Lim SC, et al. (2011) Therapeutic effect of korean red ginseng extract on infertility caused by polycystic ovaries. J Ginseng Res 35(2): 250-255.

\section{ISSN: 2574-1241}

\section{DOI: $10.26717 / B J S T R .2018 .11 .002038$}

Seok Kyo Seo. Biomed J Sci \& Tech Res

This work is licensed under Creative

Commons Attribution 4.0 License

Submission Link: https://biomedres.us/submit-manuscript.php
33. Kuang HY, Shao H, Hou LH, Wu XK (2008) Effects of ginseng total saponins on nerve growth factor expression in rat with estradiol valerate-induced polycystic ovaries. Zhong Xi Yi Jie He Xue Bao 6(7): 725-728.

34.Zhu L, Ji Li, Nannan Xing, Dongwei Han, Haixue Kuang, et al. (2015) American ginseng regulates gene expression to protect against premature ovarian failure in rats. Biomed Res Int p. 8.

35. Ge P, Xing N, Ren Y, Zhu L, Han D, et al. (2014) Preventive effect of American ginseng against premature ovarian failure in a rat model. Drug Dev Res 75(8): 521-528.

36. Yu WJ, Lee BJ, Nam SY, Yang DC, Yun YW, et al. (2003) Modulating effects of Korean ginseng saponins on ovarian function in immature rats. Biol Pharm Bull 26(11): 1574-1580.

37. Lee SR, Kim MR, Yon JM, Baek IJ, Lee BJ, et al. (2008) Effects of ginsenosides on organogenesis and expression of glutathione peroxidase genes in cultured rat embryos. J Reprod Dev 54(3): 164-170.

38. Wiklund IK, Mattsson LA, Lindgren R, Limoni C (1999) Effects of a standardized ginseng extract on quality of life and physiological parameters in symptomatic postmenopausal women: a double-blind, placebo-controlled trial. Swedish Alternative Medicine Group. Int J Clin Pharmacol Res 19(3): 89-99.

39. Kim SY, Seo SK, Choi YM, Jeon YE, Lim KJ, et al. (2012) Effects of red ginseng supplementation on menopausal symptoms and cardiovascular risk factors in postmenopausal women: a double-blind randomized controlled trial. Menopause 19(4): 461-466.

40. Koren G, Randor S, Martin S, Danneman D (1990) Maternal ginseng use associated with neonatal androgenization. Jama 264(22): 2866.

41. Zhang WY, H Teng, Y Zheng (1994) Ginseng saponin treatment for intrauterine growth retardation. Zhonghua Yi Xue Za Zhi 74(10): 608610.

42. Haron MH (2013) Modulation of ethanol toxicity by Asian ginseng (Panax ginseng) in Japanese ricefish (Oryzias latipes) embryogenesis. Comp Biochem Physiol C Toxicol Pharmacol 157(3): 287-297.

43. Lee SR, Kim MR, Yon JM, Baek IJ, Park CG, et al. (2009) Black ginseng inhibits ethanol-induced teratogenesis in cultured mouse embryos through its effects on antioxidant activity. Toxicol In vitro 23(1):47-52.

44. Holst L, Nordeng H, S Haavik (2008) Use of herbal drugs during early pregnancy in relation to maternal characteristics and pregnancy outcome. Pharmacoepidemiol Drug Saf 17(2): 151-159.

45. Liu, P, Yin H, Xu Y, Zhang Z, Chen K, et al. (2006) Effects of ginsenoside

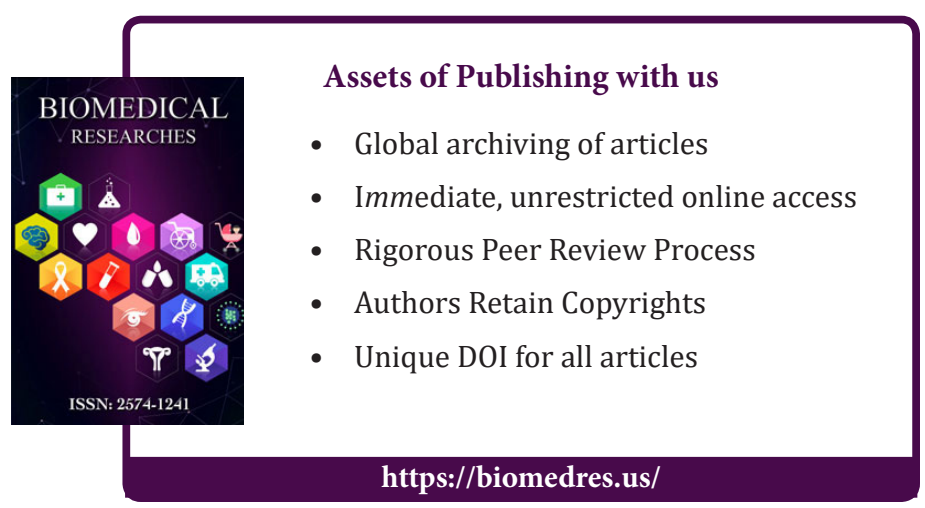

Cite this article: Bo Hyon Y, Young Sik C, SiHyun C, Byung Seok L, Si Kwan K, Seok Kyo S. Effects of Ginseng on Fertility. Biomed J Sci\&Tech Res

11(1)-2018. BJSTR. MS.ID.002038. DOI: 10.26717/ BJSTR.2018.11.002038. 